\title{
Discrimination of reproducible noise as a function of bandwidth and duration
}

\author{
THOMAS E. HANNA \\ Harvard University, Cambridge, Massachusetts
}

\begin{abstract}
The discriminability of reproducible noise bursts was studied as a function of bandwidth and duration. Listeners discriminated between trials consisting of two identical noise waveforms or two independent noise waveforms. New noise waveforms were generated each trial. In general, discrimination improved with increasing bandwidth. However, discrimination improved with increasing duration only up to about $25 \mathrm{msec}$, beyond which it decreased. Additional experiments examined discriminability with (1) forward or backward maskers, which intervened or did not intervene temporally between the comparison stimuli, (2) two noise waveforms fixed over 50 or 300 trials, and (3) high-or low-frequency noise bands. Results suggested that the decrease in discriminability beyond 25 msec was due primarily to sensory interactions of a central origin but with some effect of peripheral masking, memory interference, and attentional limitations. Information at the offset was discriminated best, and low-frequency information was better discriminated than high-frequency information. The results also indicated that envelope cues were used in certain conditions.
\end{abstract}

Auditory detection tasks provide estimates of spectral and temporal resolution that presumably reflect peripheral limitations (e.g., Green, 1973; Scharf, 1970). However, masking of a central origin has been demonstrated in several sensory modalities when the target must be identified rather than simply detected (Craig, 1982; Massaro, 1972; Turvey, 1973). Moreover, with stimuli in which information is distributed across frequency and time, nonsensory, central processes, such as attention (e.g., Watson \& Kelly, 1981), memory (e.g., Durlach \& Braida, 1969 ) and decision-making, may impose limitations on discrimination and identification performance. The encoding and discrimination of such complex stimuli as speech reflect these various limitations, which combine in ways that are not well understood. The present study examines the way in which peripheral and central factors interact to determine the discriminability of a certain class of complex auditory stimuli, that is, bursts of reproducible noise.

The discriminability of random samples of reproducible noise bursts is examined as a function of their bandwidth and duration. Increasing either the bandwidth or duration increases the amount of information that can be used to discriminate the two noise bursts. Thus, to the extent that a listener can analyze this additional information, for example, by performing short-term spectral analyses, the discrimination of two noise bursts should improve as either the bandwidth or duration is increased.

This research was supported by the National Institutes of Health. The author would like to thank S $\phi r e n$ Buus, David M. Green, Gerald Kidd, Jr., Christine R. Mason, and Donna L. Neff for their help in planning and reporting the research described in this paper. Two anonymous reviewers also provided several helpful comments. The author's mailing address is: Laboratory of Psychophysics, Harvard University, 33 Kirkland St., Cambridge, MA 02138.
If one assumes that the limitations due to central and peripheral processes are minimal, and are such that the additional spectral and temporal information is mutually independent, the largest possible improvement as bandwidth and duration are increased will be that due to statistical integration. If one assumes underlying normal distributions for the two hypotheses, "same" and "different" (or more generally that the logarithm of the likelihood ratios for each hypothesis is normally distributed), then $\mathrm{d}^{\prime}$ ought to be proportional to the square root of bandwidth and duration (Egan, 1975; Green \& Swets, 1966/1974).

Green (1960) has demonstrated such a relationship for the detection of a burst of noise in a background of noise. This result suggests that a listener is able to make use of additional spectral and temporal information in an optimal manner. However, the listener in such a task is not required to analyze or store the spectral-temporal details of the noise, because a decision can be made for a single stimuli based on the total energy within a defined band and temporal limits. The present work studies the discrimination of two noise bursts, a task that requires the analysis and comparison of two waveforms and examines whether the improvement as a function of bandwidth and duration is equal to that implied by assuming statistical integration of independent spectral and temporal analyses. If performance is inferior to that implied by optimal combination of information across frequency and time, it will be desirable to know the limitations on performance in such a task and the types of peripheral information used by the subject. Subsequent experiments examine the types of central limitations that are implied by observed performance and the types of peripheral information being used by the listener. 


\section{EXPERIMENT 1}

\section{Method}

Stimuli and Apparatus. Each noise burst was generated digitally by filling an array with independent samples from a Gaussian distribution. Stimuli were presented through a 16-bit digital-toanalog converter (DAC) at a rate of 10,000 samples/sec. Different bandwidths were obtained by filtering the output of the DAC with a series of three bandpass filters (two Krohn-Hite 3343, one General Radio 1952) or by a General Radio Model 1900-A wave analyzer (50-Hz bandwidth). The stimuli decreased at about $100 \mathrm{~dB} /$ oct for frequencies outside the passband, except for the $50-\mathrm{Hz}$ bandwidth, which decreased at about $350 \mathrm{~dB} / \mathrm{oct}$. Spectral analysis of a sample of noise verified that its spectrum was flat within the passband of the broadest filter used in this experiment. Listeners were seated in individual sound-attenuated chambers and listened diotically through TDH-50 headphones.

Four bandwidths $(50,200,800$, and $3200 \mathrm{~Hz})$ and seven durations $(0.1,0.4,1.6,6.4,25.6,102.4$, and $409.6 \mathrm{msec})$ were used. These values were chosen as ratios of four to facilitate evaluation of the predictions that performance should improve proportionally to the square root of the product of bandwidth and duration. Bandwidths are specifed in terms of 3-dB down voltages and were centered at $1000 \mathrm{~Hz}$, except for the $3200-\mathrm{Hz}$ band, which extended from 100 to $3300 \mathrm{~Hz}$. Durations are specified as the duration prior to any filtering. Spectral level of the noise bursts was $50 \mathrm{~dB}$ SPL.

Procedure. For each combination of bandwidth and duration, a two-interval, forced-choice, same-different task was used to measure the discriminability of random samples of reproducible noise. Each trial consisted of two bursts of noise separated by $500 \mathrm{msec}$, with two new waveforms generated for each trial. For "different" trials, the listener heard both waveforms. For "same" trials, the listener heard only one of the waveforms, presented twice. Feed back was given after all listeners had responded. Each of three normal listeners had $15-20 \mathrm{~h}$ of practice with a mixture of the conditions before data collection began. Data were collected in order of decreasing bandwidth, and, for a particular bandwidth, in order of increasing duration. Four sets of six 50-trial blocks were run during each 2-h session. Two consecutive sets (a total of 600 trials) used the same bandwidth and duration for the noise bursts. The first 200 trials were discarded, and results are based on the last 400 trials. The average percent correct for "same" and for "different" were converted to $\mathrm{z}$ scores, and the sum of these two $\mathrm{z}$ scores was used as an estimate of $\mathrm{d}^{\prime}$.

\section{Results and Discussion}

Figure 1 shows $\mathrm{d}^{\prime}$, averaged across subjects, as a function of duration for the four bandwidths. Standard errors were computed using the method of Gourevitch and Galanter (1967). Similar trends were observed in the data for individual listeners. Discrimination was best for durations of $25.6 \mathrm{msec}$, regardless of noise bandwidth, and was generally best for the broadest bands. A fourfold increase in bandwidth or duration never produced a doubling of $\mathrm{d}^{\prime}$ and in general produced much less. Thus, performance did not improve with increasing bandwidth or duration to the degree predicted by assuming statistical integration of information across frequency or time.

Effect of duration. The most striking discrepancy from predictions of statistical integration is that performance decreases as duration is increased beyond $25 \mathrm{msec}$. Although this decrease would be very gradual if plotted in terms of linear duration, it is significant that the additional information does not improve, much less decreases, the discriminability of the noise bursts. Given estimates of temporal resolution as small as 2-3 msec (e.g., Green, 1973), one might expect that the subject could listen to several 25-msec segments of a longer burst and do much better than for the 25-msec condition. However, increasing the stimulus duration adds energy to the stimulus that may serve to mask the discriminability of individual segments of the stimulus. It is probably inappropriate to ap-

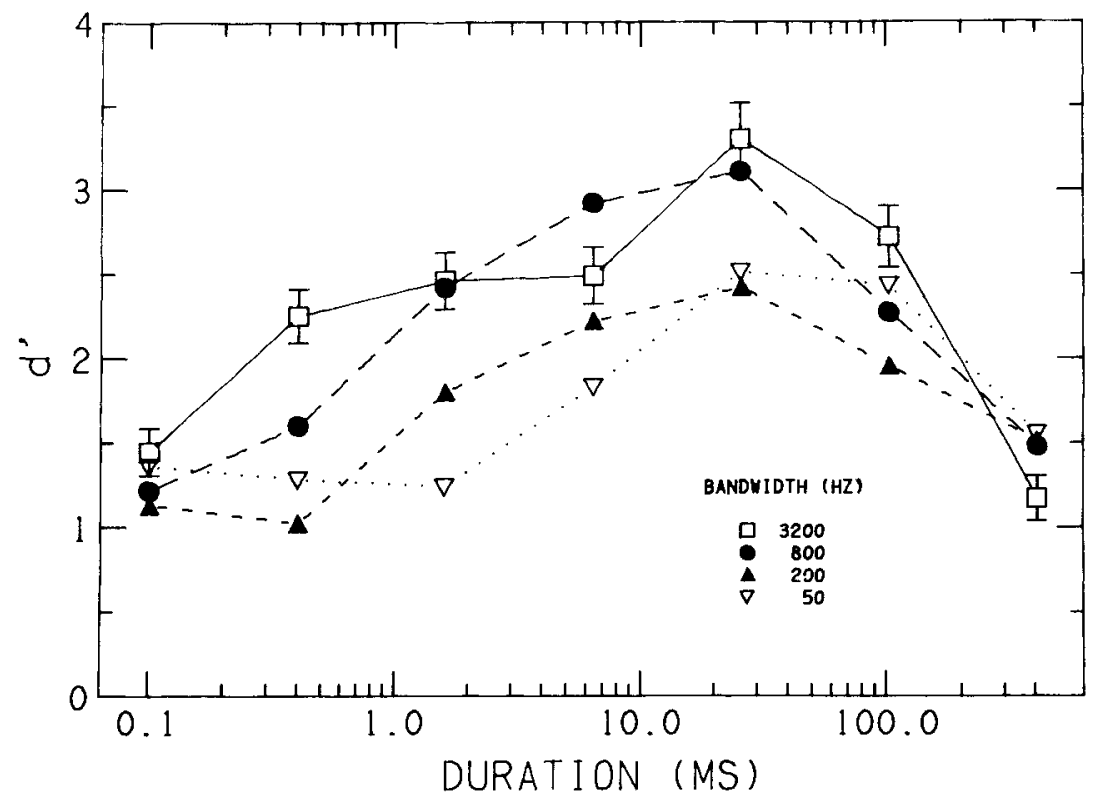

Figure 1. Average d' as a function of duration, in milliseconds, for Experiment 1. Noise bands are: 100-3300 $\mathrm{Hz}$ (squares), 600-1400 $\mathrm{Hz}$ (circles), $900-1100 \mathrm{~Hz}$ (triangles), and $975-$ $1025 \mathrm{~Hz}$ (inverted triangles). Error bars represent standard errors, averaged across listeners. 
ply time constants estimated from the detection of a signal (Penner, 1978; Penner, Robinson, \& Green, 1972) or discrimination without a masking stimulus (e.g., Patterson \& Green, 1970) to tasks that require discrimination of information subject to masking, such as the present study. Processes with longer time constants, such as a temporal integrator (e.g., Penner, 1978, 1980), adaptation (Hanna, Robinson, Shiffrin, \& Gilkey, 1982; Widin \& Viemeister, 1980), or recognition masking (Massaro, 1972), may be relatively more important for the present study.

In addition to sensory interactions, several non-sensory factors may interfere with discrimination. Durlach and Braida (1969) have outlined a theory of intensity perception which involves two distinct decision processes. One process, the trace mode, compares a relatively precise, but volatile, sensory-memory trace of the first stimulus with the second stimulus. However, this mode is ineffective (1) when the time between the two stimuli is large, because the precision of the sensory trace has decayed over time (Berliner \& Durlach, 1973; Berliner, Durlach, \& Braida, 1977), or (2) when additional stimuli intervene between the two and disrupt the sensory trace (Berliner \& Durlach, 1973). In either case, the context mode, based on an abstract and more permanent representation of the stimuli, produces better discrimination. These effects may be relevant to the present study because increasing the duration of the noise burst from 25.6 to $409.6 \mathrm{msec}$ (1) increased the interval between corresponding segments of the noise burst (e.g., the two initial 25-msec segments) allowing more time for a trace representation to decay, and (2) introduced intervening stimuli between corresponding segments of the noise burst, disrupting the trace representation. The importance of decay of the sensory trace was examined by increasing the interstimulus interval for the $25.6-\mathrm{msec}$ condition $(600-1400-\mathrm{Hz}$ band) from 500 to $884 \mathrm{msec}$, which produced an interval between onsets equal to that for the 409.6-msec condition. This increase in the interstimulus interval had only a small effect; $\mathrm{d}^{\prime}$ decreased from 3.14 to 2.97 , indicating that trace decay plays a minor role. The importance of memory interference due to intervening stimuli was examined in Experiment 2.

Other non-sensory factors that may account for poorer discrimination at longer durations include attentional effects of the form described by Watson and his colleagues (Watson \& Kelly, 1981; Watson, Kelly, \& Wroton, 1976). Their research compared discrimination of tonal sequences under conditions of high stimulus uncertainty with that under conditions of low uncertainty. Large decrements in the ability to discriminate a specific tone within the sequence was found when there was high uncertainty about the surrounding tones. This deficit could not be attributed to sensory interactions because the large deficit was not found when there was low uncertainty about the surrounding tones. Watson uses the term "informational masking" to indicate that variability in the stimulus context across trials can prevent the listener from attending to a specific temporal or spectral region. In the present experiment, increasing duration may have introduced a more variable stimulus context, which prevents the listener from attending to a specific temporal segment. Thus, discrimination of the longer bursts would not be as good as that for 25-msec bursts. The influence of a variable context was examined in Experiment 2.

Effect of bandwidth. An increase by a factor of 64 in bandwidth produces at most a doubling of performance. Although this relatively small effect of bandwidth seems to conflict with independent analysis of separate spectral regions, a possible explanation is suggested by Schacknow and Raab (1976). Bandwidths have been specified in terms of half-power frequency cutoffs. If the subject were able to use information at frequencies outside this 3-dB-down passband, the effective bandwidths may be much greater than those specified, altering the effective ratio between successive bands. The four noise bands had 20-dB-down bandwidths of $120,490,1120$, and $3725 \mathrm{~Hz}$ and 50-dB-down bandwidths of $240,855,1510$, and $4485 \mathrm{~Hz}$. These bandwidth estimates can predict the improvement of approximately square root of 2 (i.e., $(1120 / 490)^{1 / 2}$ or $\left.(1510 / 855)^{1 / 2}\right)$ observed for the $800-\mathrm{Hz}$ band relative to the $200-\mathrm{Hz}$ band for durations from 0.4 to $25.6 \mathrm{msec}$. However, these bandwidth estimates also predict that performance for the $200-\mathrm{Hz}$ band should be about a square root of 3 or 4 better than the $50-\mathrm{Hz}$ band, as should the $3200-\mathrm{Hz}$ band relative to the $800-\mathrm{Hz}$ band. Neither of these relations was observed. Thus, arguments based on effective bandwidths cannot predict the small range of performance as a function of bandwidth, although performance is predicted for bandwidths of 200 and $800 \mathrm{~Hz}$. Possible reasons for the discrepancies at the broadest and narrowest bandwidths are discussed in Experiment 3.

In Experiment 2, two subsequent sets of conditions examined factors that might serve to decrease discriminability for long-duration noise bursts. The first set of conditions (Experiment 2a) compared the effect of forward or backward maskers that either intervened or did not intervene temporally between the stimuli that were to be discriminated. These conditions tested for interference with the sensory trace (memory interference), as suggested by Durlach and Braida (1969). The second set of conditions (Experiment $2 b$ ) examined the effect of trial-to-trial uncertainty by presenting the same two noise bursts to be discriminated for an entire block. These conditions tested for an effect of uncertainty on attentional processes, as suggested by Watson et al. (1976).

\section{EXPERIMENT 2a EFFECT OF INTERVENING STIMULI}

\section{Method}

Five conditions, illustrated in Figure 2, were tested using the samedifferent procedure of Experiment 1. On each trial, two 25.6-msec bursts of bandpass noise $(600-1400 \mathrm{~Hz})$ were presented, separated by $884 \mathrm{msec}$. The waveforms were either identical or independent 


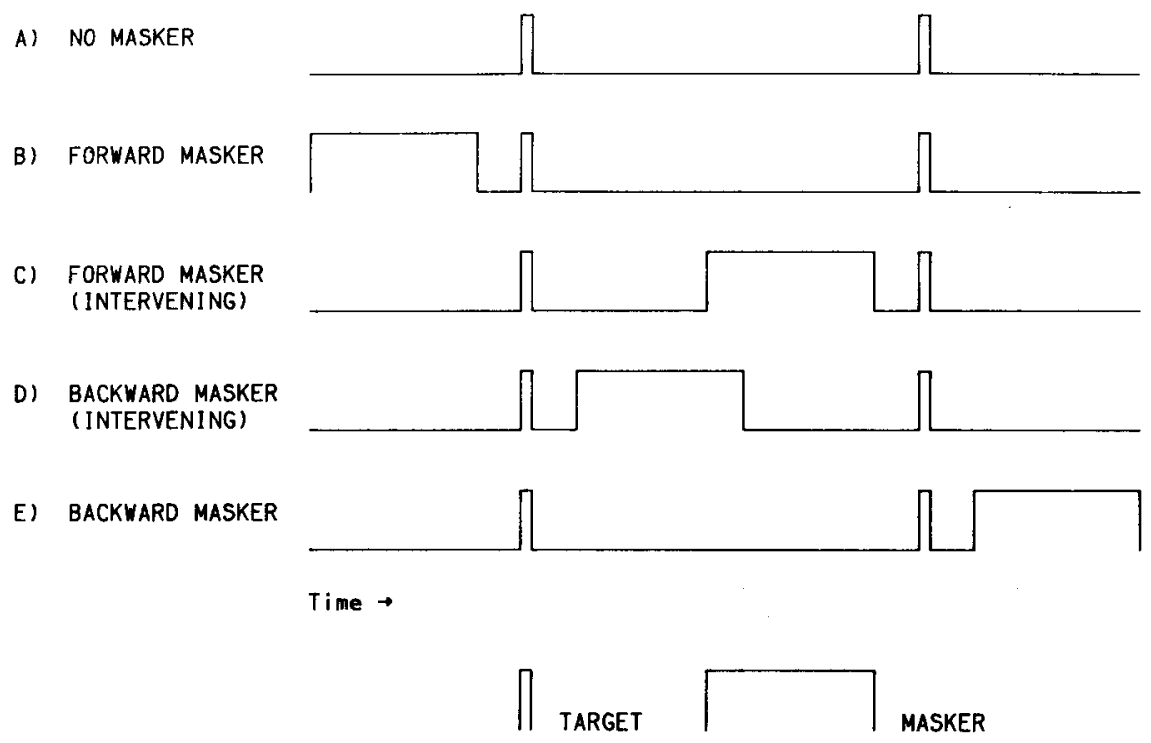

Figure 2. Schematic time line for five conditions in Experiment 2. In the top sequence, two 25.6msec bursts were presented. In the remaining four conditions, a 384-msec forward or backward masker, which either intervened or did not intervene between the two comparison stimuli, was added.

samples of noise, with new waveforms generated for each trial. In one condition, (A), only 25.6 -msec bursts were presented. In the other four conditions, an additional 384-msec burst of noise of identical bandwidth and spectral level as the 25.6 -msec stimuli occurred: (B) with its offset $100 \mathrm{msec}$ prior to the onset of Interval 1 (forward masker, not intervening), (C) with its offset $100 \mathrm{msec}$ prior to the onset of Interval 2 (forward masker, intervening), (D) with its onset $100 \mathrm{msec}$ after the offset of Interval 1 (backward masker, intervening), or (E) with its onset $100 \mathrm{msec}$ after the offset of Interval 2 (backward masker, not intervening). If the only factor limiting performance for durations in excess of $25 \mathrm{msec}$ is that due to memory interference as outlined by Durlach and Braida (1969), then performance in the two conditions without intervening stimuli (B and E) should be as good as that with no masker (A). Alternatively, if there are masking effects that are due simply to sensory interaction, then it should be irrelevant whether the additional 384-msec noise burst is temporally intervening or nonintervening between the comparison stimuli. Specifically, the forward masking conditions, B and C, should yield similar performance, as should the backward masking conditions, D and E. Results were based on 300 trials per listener for the condition without a masker and 600 trials per listener for the conditions with a masker. The conditions with a masker were run in two counterbalanced orders. The listeners were the same three that had served in Experiment 1. ${ }^{1}$

\section{Results and Discussion}

Two listeners showed the same pattern of results, with all pairwise comparisons of the five conditions significant for each listener $(\mathrm{p}<.05$, Gourevitch \& Galanter, 1967). For these two listeners, average $\mathrm{d}^{\prime}$ was 2.63 without a masker, 2.03 and 1.61 for a nonintervening and an intervening forward masker, respectively, and 1.17 and 0.62 for a nonintervening and an intervening backward masker, respectively. Although masking was obtained when the masker did not intervene, there was a greater amount of masking when the masker did intervene. With either intervening or nonintervening maskers, backward maskers are more effective than forward maskers. The third listener did not show any significant differences across conditions, with the exception that a nonintervening, forward masker produced significantly worse performance than the other conditions. That this conditions should be worse than the others is not predicted by either memory interference or sensory interaction, and may be a spuriously significant result. This listener may have shown a pattern similar to that of the others had there not been a ceiling effect, that is, no differences among the conditions.

The greater masking by intervening maskers suggests that memory interference occurred. However, masking is observed with nonintervening maskers, indicating that sensory interactions also acted to decrease discriminability. Backward masking had more of an effect than either forward masking or interference, inasmuch as discriminability with a nonintervening, backward masker $\left(\mathrm{d}^{\prime}=1.17\right)$ was worse than that with an intervening, forward masker $\left(d^{\prime}=1.61\right)$. The fact that backward maskers were more effective than forward maskers conflicts with most results for the detection of targets, which is presumably limited by peripheral filtering and adaptation (e.g., Duifhuis, 1973), but agrees with results in various sensory modalities for identification of targets, which involves masking of a more central origin (Craig, 1982; Massaro, 1972; Turvey, 1973).

\section{EXPERIMENT 2b EFFECT OF STIMULUS UNCERTAINTY}

\section{Method}

At the beginning of each 50-trial block, two 25.6-msec noise waveforms were generated. An additional 384-msec noise was 
generated and appended to each of the two 25.6 -msec bursts, resulting in two 409.6-msec noises which differed only over a $25.6-\mathrm{msec}$ target. The location of the target was the initial, middle, or final segment of the burst. Stimuli were bandpass filtered from 600 to $1400 \mathrm{~Hz}$. One of these two waveforms was selected and presented in the first interval of every trial. On "same" trials, the second interval also contained this waveform; on "different" trials, the second interval contained the other waveform. Approximately 14 blocks were run for each target condition. If the discriminability of individual segments of the burst were decreased by trial-to-trial variability, performance should improve over the course of the 50trial block with fixed stimuli.

To minimize uncertainty further, two additional conditions were run in which the two noise waveforms were fixed over a set of six 50-trial blocks. The targets were located at either the beginning or the end of the burst. Five sets were run for each condition, each set using a new selection of noise bursts. The listeners were the same three who had served in Experiment 1.

\section{Results and Discussion}

Figure 3a (left panel) shows results of the three conditions for which the noise bursts were constant for 50 trials. Percent correct for "same" and "different," combined across blocks, was computed for successive sets of 10 trials (i.e., Trials 1-10, 11-20, etc.). Each point is the average d' across listeners, with each listener's d' based on approximately 140 responses (10 trials for each of 14 blocks). ${ }^{2}$ Figure $3 \mathrm{~b}$ (right panel) shows results for stimuli fixed over 300 trials. Percent correct for "same" and "different," combined across the five sets, were computed for successive sets of 50 trials. Each point is the average d' across listeners, with each listener's d' based on 250 responses. Because of the small number of trials and noise samples, individual data were variable, but there were no apparent differences across listeners in the effects of trial block or target location. The effect of trial block was not significant for any listener for any condi- tion, although each listener tended to show improvement over the first 50 trials for targets located at the end. Performance on the last 10 trials for the data shown in Figure $3 \mathrm{a}$ and the last 50 trials for the data shown in Figure $3 \mathrm{~b}$ were significantly different from performance on a previous 25.6 -msec condition $\left(\mathrm{d}^{\prime}=2.78\right)$, except for one subject, who showed no significant difference when the target was located at the end. In general, there is little evidence for large effects of trial-to-trial variability on performance, except perhaps for targets located at the end. ${ }^{3}$

Better discrimination for targets at the end of the noise burst is consistent with the results of Experiment 2a, which showed more backward than forward masking. Targets at the end may also show an attentional benefit with low uncertainty. The finding that the functions in Figure 3 are flat suggests that informational masking does not play a large role in determining the discriminability of the noise bursts. In particular, since all conditions asymptote at a lower level of performance than for isolated $25.6-\mathrm{ms}$ bursts, informational masking is not the reason for decreased performance beyond $25 \mathrm{msec}$ in Experiment 1. It may be argued that the listeners did not receive sufficient exposure to the stimuli for the attentional effects of stimulus context to be reduced, and that with thousands of trials performance would equal that for brief bursts in isolation. However, some studies have observed effects of reduced uncertainty within 300 trials (e.g., Watson \& Kelly, 1981, Figure 7), and further evidence that the results of the present study cannot be explained in terms of informational masking can be found in Experiment 2a. Watson et al. (1976) argued that, with informational masking, introducing a 80-120-msec gap in a tonal sequence makes the component prior to the gap as discriminable as the final component. In Experiment 2a, however, backward maskers were more effective than for-

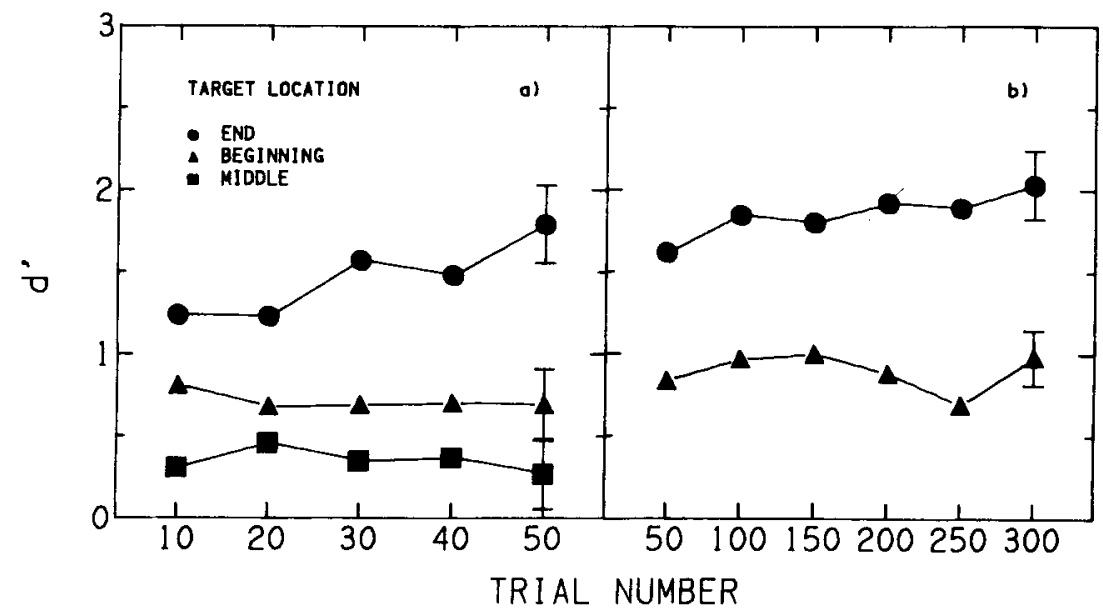

Figure 3. Average d' as a function of trial number under conditions of reduced uncertainty, for targets at the beginning (triangles), middle (squares), or end (circles). The left panel shows results for noise samples constant over a 50-trial block; the right panel shows results for noise samples constant over a set of six 50-trial blocks. Error bars are as in Figure 1. 
ward maskers even with a 100 -msec gap. The failure to find attentional effects in the present study may be due to the use of broadband stimuli, which presumably exhibit much greater sensory interaction than the tone sequences used by Watson. Nonetheless, one must still admit the possibility that attentional effects may be observed with more extensive training, particularly in light of the many differences between noise bursts and tonal sequences. Noise bursts may be more difficult to learn than tonal sequences.

The results of Experiments $2 a$ and $2 b$ suggest that sensory interactions are the primary factor for limiting the improvement, observed in Experiment 1, with increases of stimulus duration. The fact that both Experiment 2a and Experiment $2 b$ show greater backward masking than forward masking is evidence that this sensory interaction is central in origin. These experiments also demonstrated several other effects that limit performance, that is, forward masking, possibly due to peripheral interaction, memory interference, and attentional limitations, although these effects were smaller.

\section{EXPERIMENT 3 EFFECT OF CENTER FREQUENCY}

The small effect of bandwidth in Experiment 1 could be explained in part by assuming effective bandwidths broader than the 3-dB-down bandwidths. However, this characterization failed to explain why performance was relatively poorer than predicted with broad bands and relatively better than predicted with very narrow bands. Predictions for broad bandwidths assume that the listener is able to use information in the various frequency regions equally well. There are reasons to think that such an assumption is not warranted. Spectral resolution is relatively poorer and temporal resolution relatively better at high than at low frequencies (Fitzgibbons, 1983; Shailer \& Moore, 1983). Also, phase-locking of eight-nerve fibers is better at lower frequencies (Rose, Brugge, Anderson, \& Hind, 1967). Relative performance for high- vs. lowfrequency noise bands should distinguish whether spectral resolution and phase-locking or temporal resolution is more important for the discrimination. Moreover, if the temporal resolution of auditory filters determines the durations for which the masking effects observed in Experiment 2 begin to take effect, maximal performance should occur at shorter durations for high - than for lowfrequency noise bands. Narrowband noise bursts were also used to examine the possible use of envelope cues, such as pitch, roughness, or beats (Plomp, 1976), which would explain the relatively good performance with narrow bandwidths.

\section{Method}

Two of the three listeners from the previous experiments participated in this experiment. The method was identical to that of Experiment 1 , except for the bandwidths and durations of the noise bursts. The five noisebands used were: broadband $(100-3300 \mathrm{~Hz})$;
$500-\mathrm{Hz}$ wide, low-frequency $(100-600 \mathrm{~Hz})$ and high-frequency $(2800-3300 \mathrm{~Hz})$; and $50-\mathrm{Hz}$ wide, low-frequency $(225-275 \mathrm{~Hz})$ and high-frequency $(2975-3025 \mathrm{~Hz})$. Each bandwidth was used at each of five durations: $1.6,6.4,25.6,102.4$, and $409.6 \mathrm{msec}$.

\section{Results and Discussion}

Figure 4 shows $d^{\prime}$ as a function of duration for the five bandwidths. Performance improved up to durations of $25.6 \mathrm{msec}$ for all noise bands. With some exceptions, relative performance across noise bands was ordered (best to worst): broadband; low-frequency, $500-\mathrm{Hz}$ band; lowfrequency, $50-\mathrm{Hz}$ band; high-frequency, $50-\mathrm{Hz}$ band; and high-frequency, $500-\mathrm{Hz}$ band. The most notable exception occurred at $409.6 \mathrm{msec}$, where discrimination for the $50-\mathrm{Hz}$ bands exceeded that for the broader bands.

Performance at high and low frequencies was similar with $50-\mathrm{Hz}$ bands, in spite of the differences in spectral resolution and phase-locking at these frequencies. This similarity suggests the use of envelope cues for these narrow bands, for which the envelope is well defined. Slightly better performance at the lower frequency is consistent with the results of Buus (1983), who showed that envelope variation is discriminated better at lower frequencies. Increasing the bandwidth of the noise had different effects at high and low frequencies. At low frequencies, performance improved, possibly due to the use of either the spectral profile or the temporal fine-structure. At high frequencies, performance worsened, due to the lack of either of these cues and the elimination of the envelope cue. Thus, the better temporal resolution at high frequencies does not appear to aid the discrimination. Similar per-

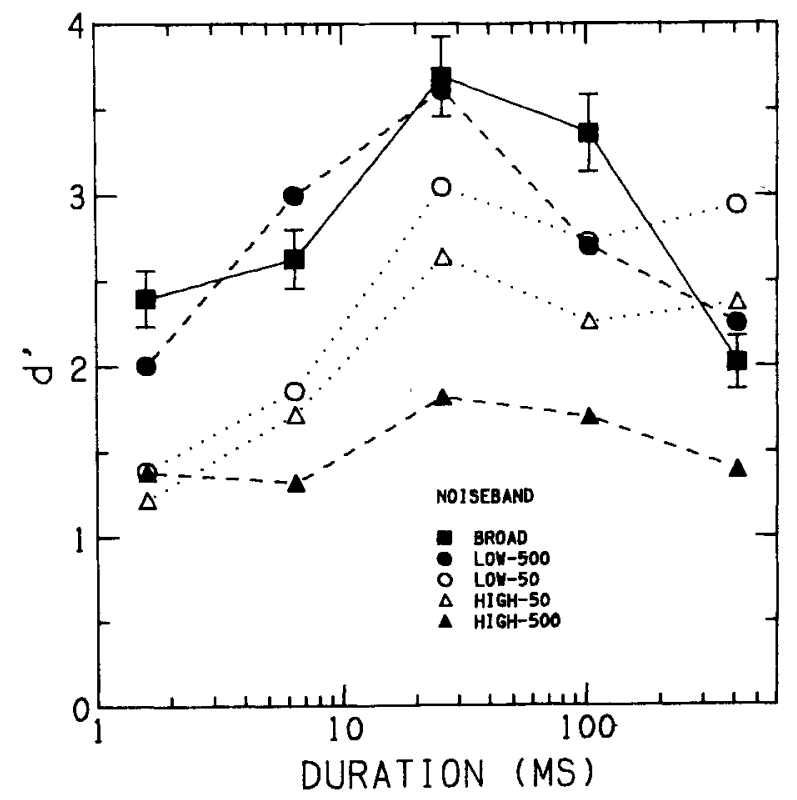

Figure 4. Average d' as a function of duration, in milliseconds, for Experiment 3. Noise bands are: 100-3300 $\mathrm{Hz}$ (squares), 100$600 \mathrm{~Hz}$ (filled circles), 225-275 Hz (open circles), 2975-3025 Hz (open triangles), and $2800-3300 \mathrm{~Hz}$ (filled triangles). Error bars are as in Figure 1. 
formance for the $500-\mathrm{Hz}$, low-frequency band and the broadest band indicates that the low frequencies were determining the discriminability of the broadband bursts. Although the finding that low-frequency information was more discriminable may be determined largely by the choice of stimuli, Green and Mason (in press) have shown that spectral shape is discriminated better at lower frequencies for five-tone complexes with components logarithmically spaced in frequency.

The finding that all of the functions in both Figure 4 and Figure 1, increase only up to durations of $25.6 \mathrm{msec}$ suggests that the masking effects studied in Experiment 2 are not determined by constraints of auditory filters, but rather by more central processes. Performance beyond $25 \mathrm{msec}$ does not decrease as much for narrow bands as for broad bands, indicating that envelope cues such as pitch, roughness, or beats may not be masked in the same way as the spectral or temporal cues that determine performance for the broader bands.

\section{SUMMARY}

Large changes in bandwidth and duration had only a small effect on the discriminability of bursts of reproducible noise. Discrimination was best for a duration of about $25 \mathrm{msec}$ regardless of bandwidth or center frequency of the noise bursts, except perhaps for $50-\mathrm{Hz}$ bandwidths. The importance of masking of a central origin was demonstrated by the fact that the effect of backward masking was greater than that of forward masking, and by the invariance of the 25-msec $p$ zak as bandwidth and center frequency of the noise bursts were varied. However, forward masking, memory interference, and attentional limitations also limited performance at the longer durations. Low-frequency information was more useful, perhaps due to better spectral resolution or phase-locking at lower frequencies. Finally, envelope cues may be important for the discrimination of narrowband stimuli. Although this aspect of performance was not studied in detail, high- and low-frequency performance was more comparable with narrowband stimuli and temporal masking effects may have been reduced. The results suggest that auditory information for discrimination and identification may be most effectively transmitted at the stimulus offset and that the stimuli should either be brief $(25 \mathrm{msec})$ and low frequency or provide envelope cues.

\section{REFERENCES}

Berliner, J. E., \& Durlach, N. I. (1973). Intensity perception. IV. Resolution in roving-level discrimination. Journal of the Acoustical Society of America, 53, 1270-1287.

Berliner, J. E., Durlach, N. I., \& Braida, L. D. (1977). Intensity perception. VII. Further data on roving-level discrimination and the resolution and bias edge effects. Journal of the Acoustical Society of America, 61, 1577-1585.

BuUs, S. (1983). Discrimination of envelope frequency. Journal of the Acoustical Society of America, 74, 1709-1715.

CraiG, J. C. (1982). Vibrotactile masking: A comparison of energy and pattern maskers. Perception \& Psychophysics, 31, 523-529.

DuifHuis, H. (1973). Consequences of peripheral filter selectivity for nonsimultaneous masking. Journal of the Acoustical Society of America, 54, 1471-1488.

DURLACH, N. I., \& Braida, L. D. (1969). Intensity perception. I. Preliminary theory of intensity resolution. Journal of the Acoustical Society of America, 46, 372-383.

EGAN, J. P. (1975). Signal detection theory and ROC analysis. New York: Academic Press.

FitzGibions, P. J. (1983). Temporal gap detection in noise as a function of frequency, bandwidth, and level. Journal of the Acoustical Society of America, 74, 67-72.

Gourevitch, V., \& Galanter, E. (1967). A significance test for one parameter isosensitivity functions. Psychometrika, 32, 25-33.

GREEN, D. M. (1958). Detection of multiple component signals in noise. Journal of the Acoustical Society of America, 30, 904-911.

GreEN, D. M. (1960). Auditory detection of noise signal. Journal of the Acoustical Society of America, 32, 121-131.

GreEN, D. M. (1973). Minimum integration time. In A. R. Moller (Ed.), Basic mechanisms of hearing. New York: Academic Press.

GREEN, D. M., \& MASON, C. R. (in press). Auditory profile analysis: Frequency, phase, and Weber's law. Journal of the Acoustical Society of America.

Green, D. M., \& Swets, J. A. (1974). Signal detection theory and psychophysics. Huntington, NY: Krieger. (Originally published, 1966)

Hanna, T. E., Robinson, D. E., Shiffrin, R. M., \& Gilkey, R. H. (1982). Forward masking of diotic and dichotic clicks by noise. Journal of the Acoustical Society of America, 72, 1171-1177.

MASSARO, D. W. (1972). Preperceptual images, processing time, and perceptual units in auditory perception. Psychological Review, 79, 124-145.

Patterson, J. H., \& Green, D. M. (1970). Discrimination of transient signals having identical energy spectra. Journal of the Acoustical Society of America, 48, 894-905.

PenNer, M. J. (1978). A power law transformation resulting in a class of short-term integrators that produce time-intensity trades for noise bursts. Journal of the Acoustical Society of America, 63, 195-201.

PenNer, M. J. (1980). Evidence for two temporal processes in forward masking. Journal of the Acoustical Society of America, 68, 455-457.

Penner, M. J., Robinson, C. E., \& Green, D. M. (1972). The critical masking interval. Journal of the Acoustical Society of America, 52, 1661-1668.

Plomp, R. (1976). Aspects of tone sensation. New York: Academic Press.

Rose, J. E., Brugge, J. F., Anderson, D. J., \& Hind, J. E. (1967). Phase-locked response to low-frequency tones in single auditory nerve fibers of the squirrel monkey. Journal of Neurophysiology, 30, 769-793.

SChaCkNOW, P. N., \& RAAB, D. H. (1976). Noise-intensity discrimination: Effects of bandwidth conditions and mode of masker presentation. Joumal of the Acoustical Society of America, 60, 893-905.

Scharf, B. (1970). Critical bands. In J. V. Tobias (Ed.), Foundations in modern auditory theory (Vol. 1). New York: Academic Press.

Shailer, M. J., \& Moore, B. C. J. (1983). Gap detection as a function of frequency, bandwidth, and level. Journal of the Acoustical Society of America, 74, 467-473.

Swets, J. A., Shipley, E. F., McKey, J. J., \& Green, D. M. (1959). Multiple observations of signals in noise. Journal of the Acoustical Society of America, 31, 514-521.

TuRVEY, M. T. (1973). On peripheral and central processes in vision: Inferences from an information-processing analysis of masking with patterned stimuli. Psychological Review, 80, 1-52.

WATSON, C. S., \& KELLY, W. J. (1981). The role of stimulus uncertainty in the discrimination of auditory patterns. In D. Getty \& J. Howard (Eds.), Auditory and visual pattern recognition. Hillsdale, NJ: Erlbaum.

Watson, C. S., Kelly, W. J., \& Wroton, H. W. (1976). Factors in the discrimination of tonal patterns. II. Selective attention and learning under various levels of stimulus uncertainty. Journal of the Acoustical Society of America, 60, 1176-1186.

WIDIN, G. P., \& VIEMEISTER, N. F. (1980). Masker interaction in puretone forward masking. Journal of the Acoustical Society of America, 68, $475-479$. 


\section{NOTES}

1. The 100 -msec gap was introduced because the task was quite difficult without any gap. The restricted range would have precluded any meaningful comparisons across conditions. This result by itself is probably indicative that either sensory interaction or informational masking is of primary importance. Any sensory masking effects observed with a 100 -msec gap would presumably be even larger for the stimuli used in Experiment 1 where no gap was present.

2. The d's were also computed for each sample of noise for the block of 50 trials. The within-subject variability of $\mathrm{d}^{\prime}$ for each condition was such that the mean-to-sigma ratio (i.e., mean $\mathrm{d}^{\prime}$ divided by the standard deviation of $d^{\prime}$ ) was in the range 1-2. The larger values of mean to sigma were obtained for larger mean values of $d^{\prime}$.
3. A minimal uncertainty condition was run in which the entire 409.6 msec was the "target," that is, at the beginning of each 50-trial block, two independent 409.6-msec noise bursts were selected. Average $\mathrm{d}^{\prime}$ was 2.59 . This value is comparable to that predicted by statistical summation of the d's from individual 25 -msec segments as estimated by the results of Experiment $2 \mathbf{b}$. Other data, for conditions with new waveforms selected on each trial, also indicate that the combination of information across segments is nearly optimal. This result has beenfound in previous psychophysical experiments (e.g., Green, 1958; Swets, Shipley, McKey, \& Green, 1959), and indicates that this aspect of decision-making does not impose a limitation on performance.

(Manuscript received April 16, 1984; revision accepted for publication October 15, 1984.) 